\title{
\Genome Medicine
}

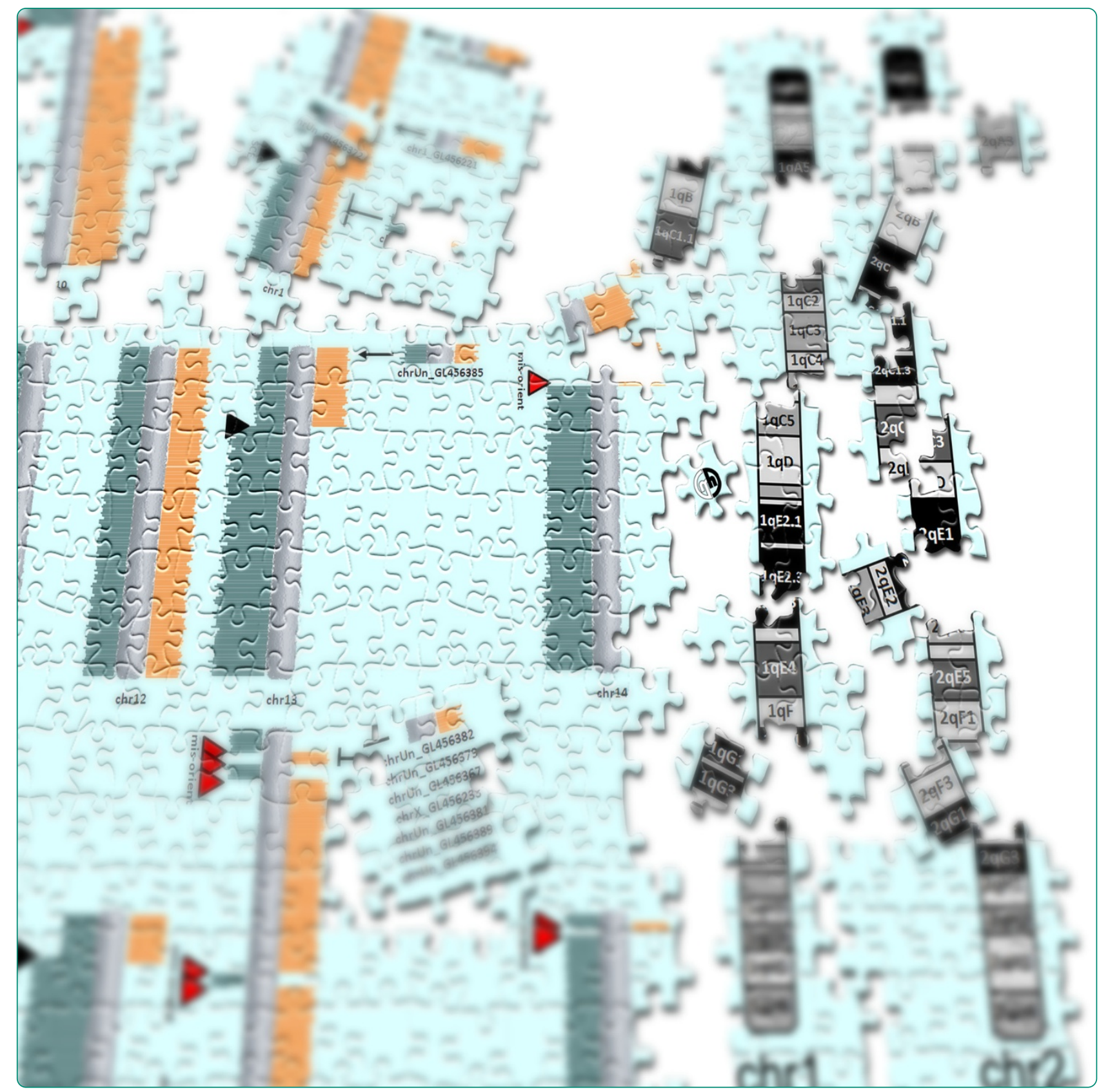

\section{BAIT: Organizing genomes and mapping rearrangements in single cells}

Hills et al. 


\title{
BAIT: Organizing genomes and mapping rearrangements in single cells
}

\author{
Mark Hills ${ }^{1 *}$, Kieran O'Neill ${ }^{1}$, Ester Falconer ${ }^{1}$, Ryan Brinkman ${ }^{1}$ and Peter M Lansdorp ${ }^{1,2,3^{*}}$
}

\begin{abstract}
Strand-seq is a single-cell sequencing technique to finely map sister chromatid exchanges (SCES) and other rearrangements. To analyze these data, we introduce BAIT, software which assigns templates and identifies and localizes SCEs. We demonstrate BAIT can refine completed reference assemblies, identifying approximately $21 \mathrm{Mb}$ of incorrectly oriented fragments and placing over half (2.6 Mb) of the orphan fragments in mm10/GRCm38. BAIT also stratifies scaffold-stage assemblies, potentially accelerating the assembling and finishing of reference genomes. BAIT is available at http://sourceforge.net/projects/bait/.
\end{abstract}

\section{Background}

We recently described a sequencing technique called Strand-seq for directional sequencing of DNA template strands in single cells $[1,2]$. To generate Strand-seq data, cells are cultured with the thymidine analogue 5-bromo2 '-deoxyuridine (BrdU) for one round of DNA replication. The newly formed DNA strands incorporate BrdU, and are selectively removed prior to library amplification, resulting in directional libraries consisting of only template strands. Sequencing of these libraries on an Illumina platform results in reads that map either to the 'Crick' strand (plus or top strand) or the 'Watson' strand (minus or bottom strand) of the reference genome. Because most eukaryotic genomes are diploid, the template strands from both chromosomal copies are represented, and the resultant directional reads can be output in the form of a chromosome ideogram (Figure 1a). Thus if a chromosome has reads mapping solely to the Watson strand, the cell has inherited a Watson template from each of the parental homologues (WW), whereas if it has reads mapping to both Watson and Crick, the cell has inherited one Crick-template and one Watsontemplate parental homologue (WC). This ability to discern which template strands were inherited by dividing cells can be used for a number of important applications including the high-resolution mapping of SCEs, analysis

\footnotetext{
* Correspondence: mhills@bccrc.ca; p.m.lansdorp@umcg.nl

${ }^{1}$ Terry Fox Laboratory, BC Cancer Agency, Vancouver, BC V5Z 1L3, Canada ${ }^{2}$ Division of Hematology, Department of Medicine, University of British Columbia, 675 West 10th Avenue, Vancouver, BC V5Z 1L3, Canada Full list of author information is available at the end of the article
}

of genomic rearrangements, and refining of reference assemblies.

SCEs are the outcome of the repair of double strand breaks, and their accumulation is an early indicator of genomic instability [3]. Strand-seq data allows the identification and mapping of these events at unprecedented resolution [1]. The frequency of SCEs has been used as a surrogate for assessing the toxicity of mutagens [4], and as a diagnostic marker for disorders such as Bloom's syndrome, which have a characteristically high frequency of SCEs [5]. Stand-seq can also detect translocations, inversions, deletions, and amplifications. Deletions and amplifications present as a loss or gain of reads over particular regions, and will locate to the same region across all libraries, making them easy to identify. Translocations and inversions appear identical to SCE events in individual libraries (Figure 1c), but can be resolved when the event locations are compiled across multiple libraries, as they will all occur over the same region. Preliminary data suggests that this approach works well in identifying and localizing chromosomal abnormalities (manuscript in preparation). It is further possible to apply Strand-seq to estimate the frequency of genomic rearrangements in a heterogeneous population of cells.

We showed previously that Strand-seq also has an application in correcting incorrectly oriented portions of the mouse reference assemblies [1]. Reference assemblies have become essential tools for aligning sequences and identifying variations, and thus, the need for a complete and accurate reference genome for any organism of interest is essential [6]. At present, a variety of organisms 


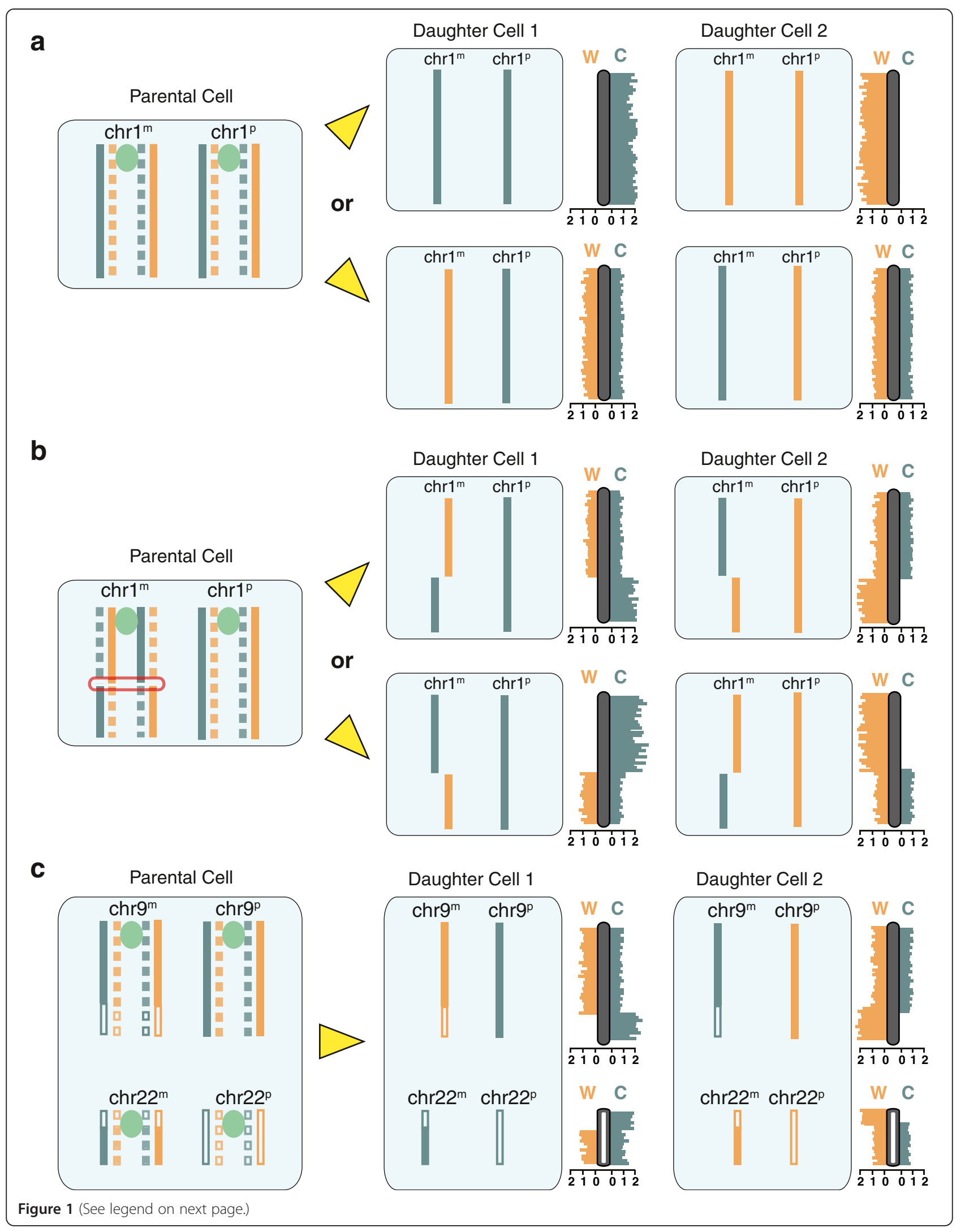




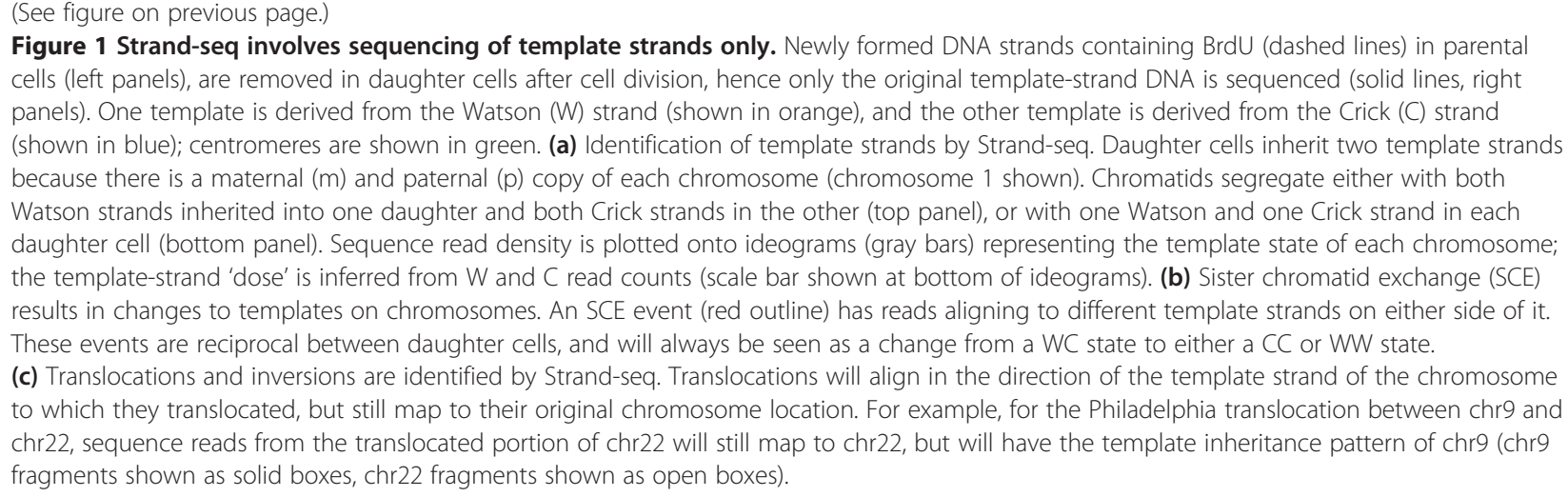

have been targeted for genome sequencing projects [7], and more established genomes are being continually updated. For example, the mouse reference genome was first published in 2002 [8], and has been periodically updated with more complete and corrected assembly versions. In most such iterations of reference assemblies, there are both gaps of unknown length within the sequence (typically regions difficult to sequence), and 'orphan scaffolds' that have yet to be mapped to particular chromosomes or regions on specific chromosomes (likely to map within gaps, and lacking the tiling to form contiguous sequences). Although PCR-based approaches [9], forms of restriction mapping $[10,11]$ and optical mapping [12] can be used to bridge these gaps or connect orphan scaffolds, there are still currently 628 gaps and 44 orphan scaffolds in the latest mouse reference assembly (GRCm38/mm10), and 357 gaps and 65 orphan scaffolds in the latest iteration of the human assembly (GRCh37/hg19). Many of the gaps are unbridged, representing spaces in the genome build of unknown length, and importantly, the relative orientation of sequences on either side of these gaps are also unknown. Furthermore, there are many early-build genome projects underway, most of which remain at the contig stage, consisting of thousands of contiguous sequences that are unplaced with respect to each other, and not localized to any chromosomes. With recent efforts aiming to rapidly generate reference genomes from 10,000 organisms $[13,14]$, the need for alternate approaches to build the thousands of contigs from scaffold-level genomes into useable reference assemblies is paramount, and here we show that Strand-seq can perform a pivotal role in this.

Strand-seq has many applications for the study of tumor heterogeneity and evolution, and for genome instability in diseases of aging, as well as an enormous potential for rapidly building and refining the growing repertoire of reference assemblies. It is also an efficient technique, with the ability to sequence up to 200 indexed libraries simultaneously on a single lane. However, in order to analyze Strand-seq features across these large datasets, the technique needed an intuitive software package that could automate this process. Here we describe new open source software, Bioinformatic Analysis of Inherited Templates (BAIT), which builds upon our previously described plotting function [1] and enables high-throughput analysis of Strand-seq data. BAIT is a command line-driven application for UNIX platforms, available under the two-clause Berkeley Software Distribution (BSD) license [15].

\section{Implementation}

\section{Data management and processing}

BAIT provides a core framework for Strand-seq analysis, including functionality to plot $\mathrm{W}$ and $\mathrm{C}$ template strands, count aneuploid chromosomes, and map and enumerate SCE events (see Additional file 1: Figure S1). Extending these core functions for genome assembly, BAIT leverages strand-inheritance data to identify misoriented contigs, localize orphan scaffolds to specific chromosome regions on late-build genomes, and assemble early-build genomes de novo from non-overlapping fragments, using only one lane of sequencing containing up to 200 indexed libraries. In concert with Strand-seq, BAIT has major applications in detecting SCEs, analyzing sister chromatid segregation, and building and finishing genome assemblies.

BAIT accepts sequencing data in BAM format and parses it with SAMtools [16] to remove duplicate reads, threshold for quality, and discern read direction. These data are then fed to multiple $\mathrm{R}$ scripts (incorporating packages from Bioconductor [17]), which bin the data (200 kb windows by default), and compute strand inheritance, perform SCE analysis and plot chromosome ideograms showing read density, directionality, and predicted SCE events (Figure 1). Additional options in the command line allow alternate forms of output, additional plotting parameters, and the ability to convert 
data into BED files that are auto-formatted for UCSC genome browser upload using the BEDtools package [18].

The ability of BAIT to accurately assess SCE events and genome build analyses can be confounded by technical variability from the Strand-seq protocol, including spurious or constant low-background reads, or variable read depths. Much of this variability is presumably engendered by BrdU uptake by the cell, and the subsequent successful removal of the BrdU-incorporated (non-template) strand from the pre-amplified library. In order to aid decisions to remove low-quality libraries from further analysis, BAIT calculates this metric by first performing an unfiltered prediction of strand inheritance, then computing library background as the average frequency of spurious non-template-strand reads (C reads on chromosomes when homozygous W template strands were inherited, and vice versa). This value is expressed as a background percentage on each library ideogram.

A summary file is also generated (see Additional file 2: Supplemental Data File 1), including the frequency of WW, WC, and CC template inheritance for each intact chromosome for the analysis of sister chromatid segregation. The distributions of template strands are presented as pie charts, showing $P$-value significance from $X^{2}$ analysis after Holm correction [19]. BAIT also plots the template inheritance across each bin of every chromosome (see Additional file 2: Supplemental Data File 1), and creates BED files of the locations of all SCE events, which is useful for all subsequent analysis of Strand-seq data, such as mapping SCEs and genomic rearrangements.

The 62 Strand-seq libraries used in this study are publically available from the Sequence Read Archive SRA055924, and have been published previously [1]. BAIT took 81 minutes to process these libraries, with an average of 3,235,111 reads each, using a single core of an Intel i7-870 $2.93 \mathrm{GHz}$ processor on a computer with 16 Gb of RAM.

\section{Detection of sister chromatid exchanges, misorientations, and genomic rearrangements}

SCEs are visualized on the chromosome ideograms as regions where reads switch from a homozygous template state (WW or CC) to a heterozygous template state (WC). Although the overall read depth is unchanged across an SCE, the proportion of directional reads will change from two copies in the homozygous state to one in the heterozygous state (Figure 1). BAIT exploits the similarity of the change in template copy number to copy number variation (CNV) analysis in order to locate and characterize all SCE events. It does this by calculating the ratio of Watson and Crick reads within each bin, using $[(\mathrm{W}-\mathrm{C}) /(\mathrm{W}+\mathrm{C})]$, and normalizing to the nearest integer. This gives a value of 1 when all reads map to the Watson strand (WW strand inheritance), -1 when all reads map to the Crick strand (CC), and 0 for an equal number of both (WC) (Figure 2a). A change in this ratio along the length of a chromosome corresponds to the location of an SCE event (Figure 2a), which is first localized to neighboring bins. For example, using the default bin size of $200 \mathrm{~kb}$, a switch from a CC templatestrand state in one bin (ratio $=-1$ ) to a WC templatestrand state in a neighboring bin $($ ratio $=0$ ) indicates that an SCE event occurred somewhere within the 400 $\mathrm{kb}$ interval encompassing those two bins (Figure 2a).

BAIT first makes gross event calls by utilizing the circular binary segmentation algorithm [20] implemented in the CNV Bioconductor package DNAcopy [21] to locate the SCE event to the two-bin interval. It then recalculates the template-strand ratio by segmenting this interval into five new bins $(80 \mathrm{~kb}$ each using default bin size), narrowing the location of the SCE interval further. BAIT applies this binning-based DNA-copy detection method iteratively, decreasing the bin size by a factor of five each time (Figure 2b), until the read density is no longer sufficient to make accurate calls (determined to be when an interval has less than 50 reads, or when DNAcopy can no longer predict a single event (Figure 2c). In order to identify SCE events on the boundary of bins, BAIT pads each interval with one-half of the interval length in each direction (Figure 2b,c; red arrows).

BAIT then refines the gross interval by incorporating a simple walker algorithm that analyzes reads starting from the homozygous state, and reports the first read on the opposite template that represents a switch to a heterozygous state (Figure 2c; green box). From this refined interval, the walker checks that the 10 preceding reads map to the homozygous state, and that at least 4 of the 20 following reads map to the opposite template state (Figure 2c). If these criteria are not met, as may be the case where the background is high, BAIT continues to analyze the across the interval until they are met. These checks improved the localization of SCE events (see Additional file 3: Figure S2), and varying these thresholds did little to change the data. Through this two-step process, BAIT automatically detects and localizes SCEs with a high degree of confidence, plots them on ideograms, and creates a UCSC-formatted BED file of all SCE event intervals.

BAIT amalgamates all called SCE events across libraries to identify any locations that have multiple SCE events associated with them. It reports any SCE-like event that occurs over the same interval in more than one library, treating them as a potential structural (genomic rearrangement) event, and calculating the number 


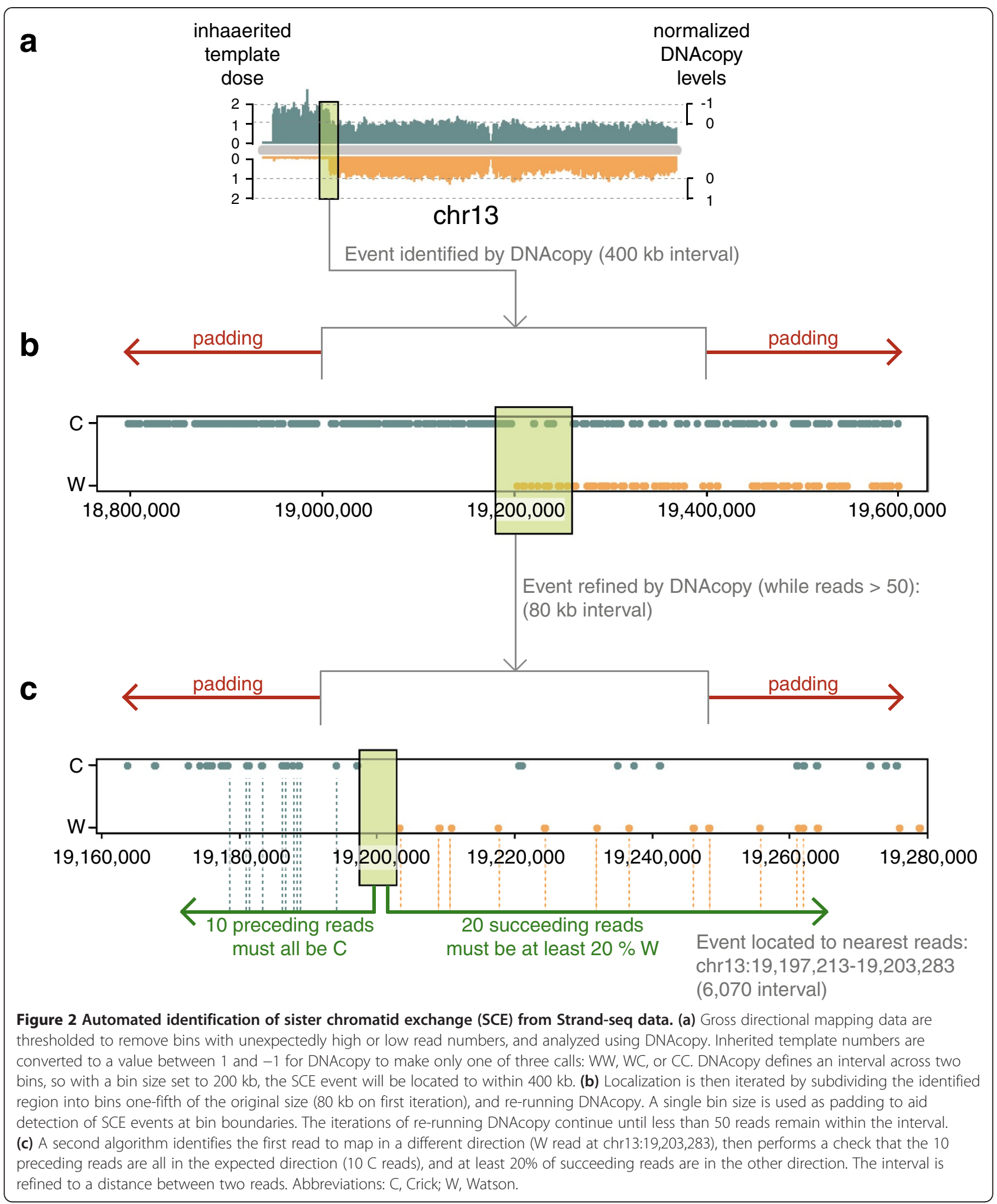

of occurrences. Events occurring in the same location over multiple libraries either are regions of recurrent SCE, or represent translocations, deletions, or inversions (Figure 1c). In addition, duplications are identified using the CNV function across each chromosome, and chromosomal anueploidy is calculated by comparing the read depth of each chromosome to the average read depth within the (diploid) library. A chromosomal read 
depth of half the library average corresponds to a single copy (monosome), whereas $1.5 \times$ the library average corresponds to three copies (triploid).

Although SCEs show a transition from a homozygous to a heterozygous template state (WW to WC, or CC to WC) in Strand-seq libraries, transitions between two homozygous template states (WW to CC and CC to WW) are identified as misoriented fragments in the reference genome. Previously, we manually identified and localized these events to unbridged gaps, and confirmed a subset of misorientations by hybridization of directional probes [1]. BAIT distinguishes these events from SCEs, and writes the locations of these data to a separate CSV file. Invariably, misorientations in the reference genome will present as a template-strand switch in every Strand-seq library, so BAIT also computes the concordance across all libraries as a measure of robustness of the misorientation call. Because BAIT already calculates chromosomal aneuploidy, an SCE event in a monosome chromosome (W to $\mathrm{C}$ or $\mathrm{C}$ to $\mathrm{W}$ ) will not be erroneously called as a misorientation (WW to CC or CC to WW).

\section{Stratification of early-build genome assemblies}

Early-build genome assemblies consist of many contigs, which are effectively unanchored and unordered. However, performing Strand-seq on cells derived from organisms with early assemblies will yield directional strand information for each contig, and any contigs residing on the same chromosome will inherit the same templates. Contigs from different chromosomes will inherit template strands independently, and by chance, the templates will be the same in only half of all libraries. Conversely, adjacent contigs will inherit the same template strands across all libraries. By comparing all contigs together, it is possible to cluster them into putative chromosomes based on the concordance between them.

BAIT initially excludes libraries where every contig has inherited WC templates (probably a failed Strandseq library), as well as individual contigs that have inherited WC templates in all libraries (probably a contig with degenerate sequences that cannot be placed). It then uses a two-stage approach to assemble the remaining contigs into a putative assembly. First, it clusters all contigs with highly similar template inheritance into linkage groups that represent individual chromosomes. It does this by comparing the two contigs represented across the most libraries, and assessing template-strand concordance between them; if they share a high concordance, they are classified together in a single linkage group, otherwise they are classified into separate linkage groups. Each remaining contig in the assembly is individually compared with the groups already assigned, and is then either added to a linkage group if it shares a high similarity with that group, or is classified into a new linkage group if it does not. This process continues until all contigs have been stratified into linkage groups or classified as single unlinked contigs. Ideally, the number of linkage groups is equal to double the number of chromosomes within the organism (a plus-strand and minus-strand linkage group for each chromosome).

To distinguish contig orientation, BAIT generates an initial contig dissimilarity matrix using only chromosomes that have inherited homozygous $\mathrm{WW}$ and CC templates (but excluding WC), in such as way that misoriented linkage groups derived from the same chromosome are highly dissimilar (Figure 3a, left panel). BAIT then uses a simple greedy algorithm to reorient the misoriented linkage groups, iteratively inverting the most dissimilar, and recomputing the distance matrix until a reorientation causes no increase in the summed concordance of all groups (Figure 3a, right panel; see Additional file 4: Figure S3). Linkage groups with high similarity are merged in the recomputed data, and BAIT visualizes this as a distance-matrix heat plot of linkage group concordance (Figure 3a, right panel; see Additional file 4: Figure S3).

The second stage in BAIT scaffolding is performed individually on each linkage group/putative chromosome, by analyzing the contigs within each group. These contigs are compared with each other, and a relative order is computed based on template-strand concordance. If a chromosome had no SCEs in any libraries analyzed, every contig from that chromosome will share an identical template-strand inheritance, and their order cannot be determined. However, because SCEs switch template-strand inheritance along chromosomes, every SCE event will switch template strands along linkage groups (LGs), and therefore stratify the contigs within it. A single SCE event will split LGs into a cluster of contigs with homozygous WW or CC template inheritance to one side of the SCE event, and a cluster of contigs with heterozygous WC templates to the other side of the SCE event. In this way, the cumulative SCEs on any particular chromosome can be compiled across all libraries to help order contigs within the LG.

Similar to how meiotic recombination is used to create a genetic linkage map between loci [22], SCE events along the chromosome can be used to determine a genetic distance between contigs on the same chromosome, allowing them to be arranged and ordered. Adjacent contigs will have a lower probability of an SCE between them and a higher chance of inheriting the same template strands across all the libraries compared with contigs at opposite ends of the chromosome, which will be far more likely to have an SCE event between them. BAIT uses template-strand inheritance and SCE 


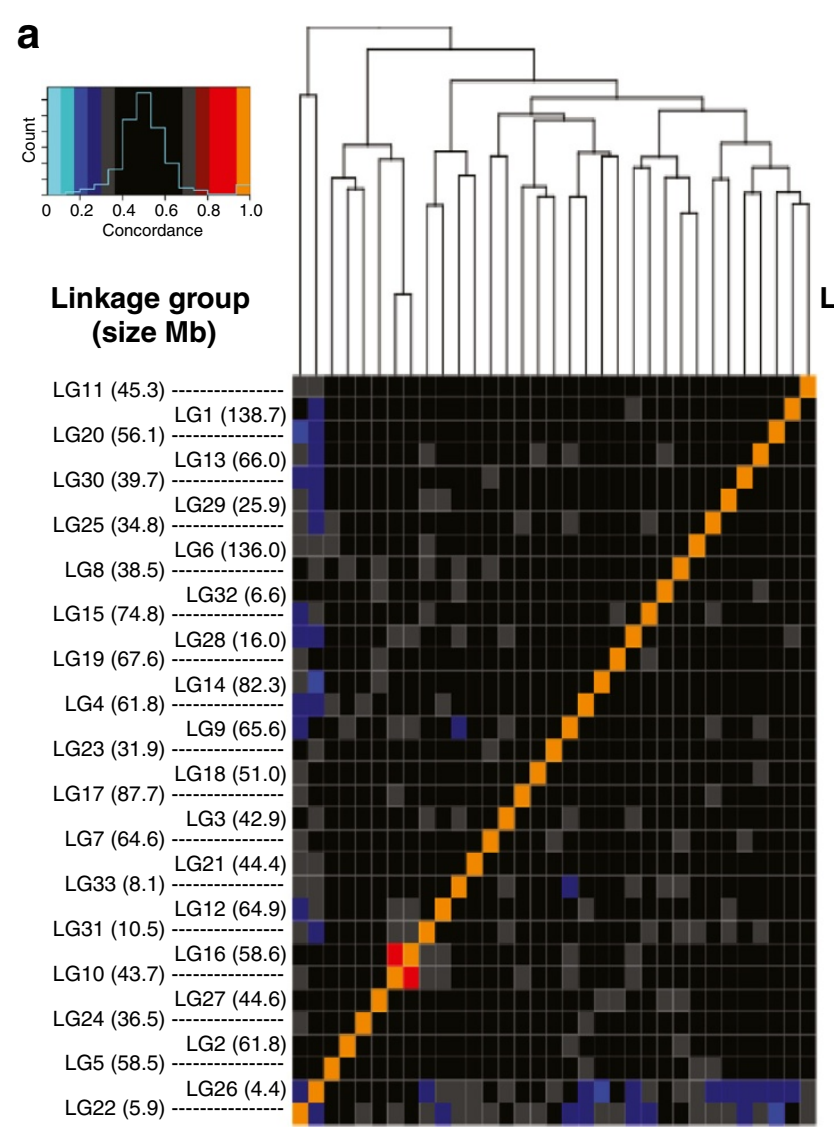

Raw clusters

Only WW and CC states counted 33 clusters $(57,581$ contigs $)$

\section{Linkage group (size Mb)}

LG13 (125.8)

LG16 (56.1)

LG11 (74.1)

LG19 (39.7)

LG14 (119.6)

LG6 (152.0)

LG7 (45.1)

LG10 (109.3)

LG20 (10.5)

LG15 (67.6)

LG9 (103.9)

LG18 (62.4)

LG2 (106.3)

LG1 (138.7)

LG5 (58.5)

LG3 (107.4)

LG4 (61.8)

LG8 (65.6)

LG12 (82.3)

LG17 (10.3)

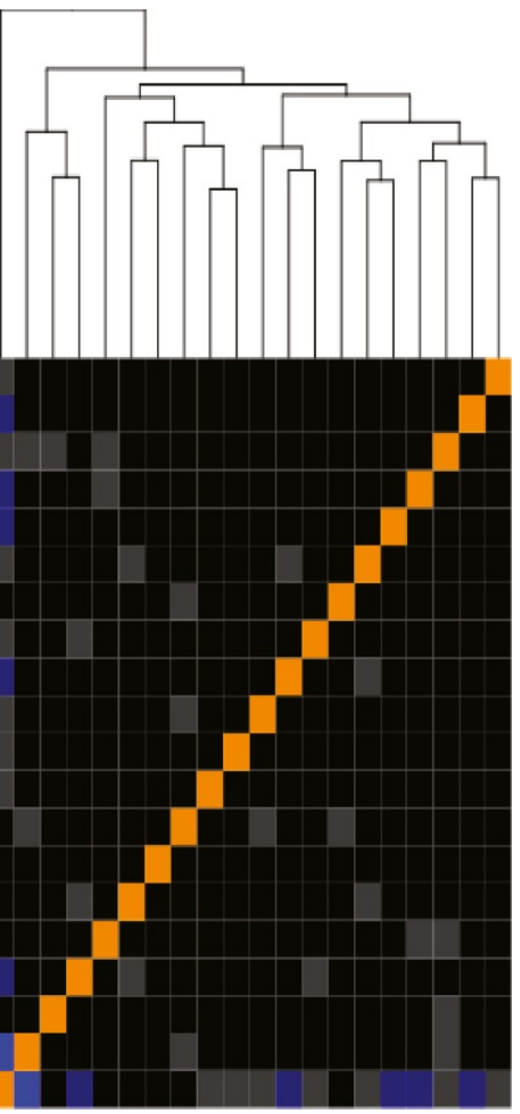

Merged and oriented clusters All states counted 20 clusters $(54,832$ contigs)

b

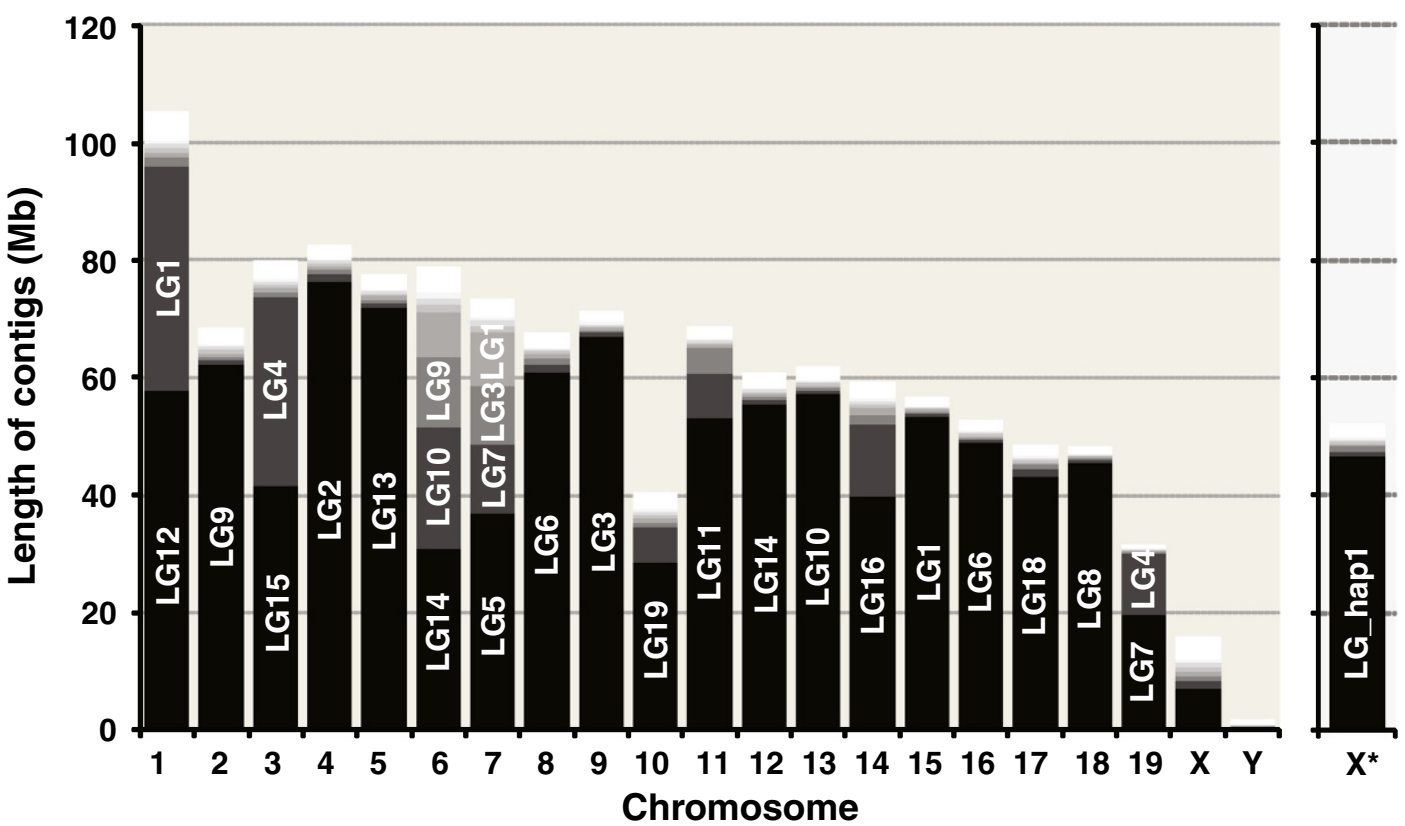

Figure $\mathbf{3}$ (See legend on next page.) 
(See figure on previous page.)

Figure 3 Clustering contigs into linkage groups for early-assembly genomes. Using template strand directionality as a unique signature, all contigs in the early mouse assembly MGSCv3 were compared with each other across all 62 Strand-seq libraries. All contigs with similar (>85\%) template inheritance patterns were stratified into linkage groups (LGs). (a) Heat plots of all BAIT-called LGs show limited similarity between groups. Through analysis of homozygous template states only (WW and CC, left panel) 57,581 contigs cluster into 33 LGs, with the association between linkage groups appearing as yellow points if groups are in the same orientation, or blue points if the groups are in opposite orientations. The LGs are then reanalyzed after merging and reorientation of associated clusters, resulting in only 20 linkage groups consisting of 54,832 contigs. (b) Histogram of the number of fragments within a linkage group that map to a particular chromosome. The LG with the largest number of contigs are shown at the bottom in dark gray, with groups that contain the next largest numbers of contigs shown in progressively lighter grays. Most LGs contain contigs that belong to the same chromosome (see Additional file 4: Figure S3), and in general, most chromosomes are represented by one or two linkage groups. Note: contigs derived from sex chromosomes in male libraries can be distinguished as they are haploid, and are not computed as an initial heat plot. Any contigs derived from haploid chromosomes are separated and clustered independently. Almost all contigs clustered into this linkage group mapped to the X chromosome (right histogram). Abbreviations: C, Crick; W, Watson.

localization to build an inter-contig distance matrix for each linkage group. Then, using a traveling salesman algorithm (similar to finding the shortest route to take for traveling to multiple destinations only once) [23], BAIT calculates the shortest path through the distance matrix on each chromosome, thereby inferring the relative order of contigs within a linkage group.

\section{Stratification of late-build genome assemblies}

Using scaffold-level and chromosome-level assemblies to generate functional reference assemblies is valuable, but it is important to note that 'completed' assemblies also contain a large number of contigs that remain unmapped. Assigning locations for these orphan scaffolds in a chromosome context is a high-priority endeavor for sequencing centers, and there are very few techniques that are available for this task [24]. However, provided that the orphan scaffold has sufficient read coverage, Strand-seq can be used to determine the strandinheritance pattern, which will be the same as the chromosome on which it is present. For example, an orphan scaffold inheriting WC template strands must locate to a WC chromosome in that particular library. If an orphan scaffold inherits WW template strands, it will locate to a WW chromosome if both sequences are in the same orientation, or to a CC chromosome if it is misoriented with respect to the chromosome. On average, using just a single library, half of the chromosomes can be excluded as possible locations for these orphan scaffolds (Figure 4a).

By comparing these locations across a batch of libraries, BAIT localizes these scaffolds to particular chromosomes. For each orphan scaffold with sufficient reads, BAIT assigns a template state, compares this against the template state of each chromosome within a particular library, and then iterates this process to compute the concordance across all libraries. Concordance is never $100 \%$ in practice, owing to libraries with high background, orphan scaffolds with too few reads to accurately call strands, SCE events within gaps between the scaffolds, and the 5 to $10 \%$ error rate of BAIT in SCE detection. Nevertheless, BAIT is still able to achieve high-quality predictions of scaffold location by taking the highest-concordance chromosome. Chromosomes are further split based on SCE locations, allowing for localization of orphan scaffolds to particular chromosomal regions (Figure 4). Because orphan scaffolds are likely to be located within gap regions rather than within contiguous sequence, BAIT can use a provided BEDformat gap file to cross-reference all mapped orphan scaffold locations to gaps within the same interval. BAIT outputs in a BED file both the best predicted region for each fragment and any candidate gaps within that region.

\section{Results and discussion}

\section{Accurate localization and mapping of SCES}

To assess the ability to computationally identify SCE events, BAIT predictions were compared with 528 SCE events from 62 murine embryonic stem cell Strand-seq libraries that had previously been identified manually [1]. Manual processing of SCE events involved uploading BED-formatted Strand-seq data into the UCSC genome browser [25], and identifying the interval at which the templates switch. Initial comparisons showed that although BAIT identified over 97\% of SCEs called manually, it also displayed a high false-discovery rate. To reduce this rate, a user-changeable threshold was incorporated, which excludes any bins that deviate from the average read depth, and thus have fewer or greater reads than expected.

By comparing the BAIT SCE calling to the manually processed SCEs, we found the optimal threshold for these data was to exclude bins with read counts of \pm 0.2 standard deviations from the mean, which gave a sensitivity of 0.93 (10.9\% false positives), and a specificity of 0.89 (7.2\% false negatives) (Figure $5 \mathrm{a}$ ). When only those libraries with a low background metric $(<5 \%)$ were included, the specificity improved to 0.94 , while the sensitivity remained almost the same at 0.92 (Figure $5 \mathrm{~b}$ ). Of the false-negative calls, $72.9 \%$ were SCEs within $5 \mathrm{Mb}$ of the start or end of the chromosome, indicating that 

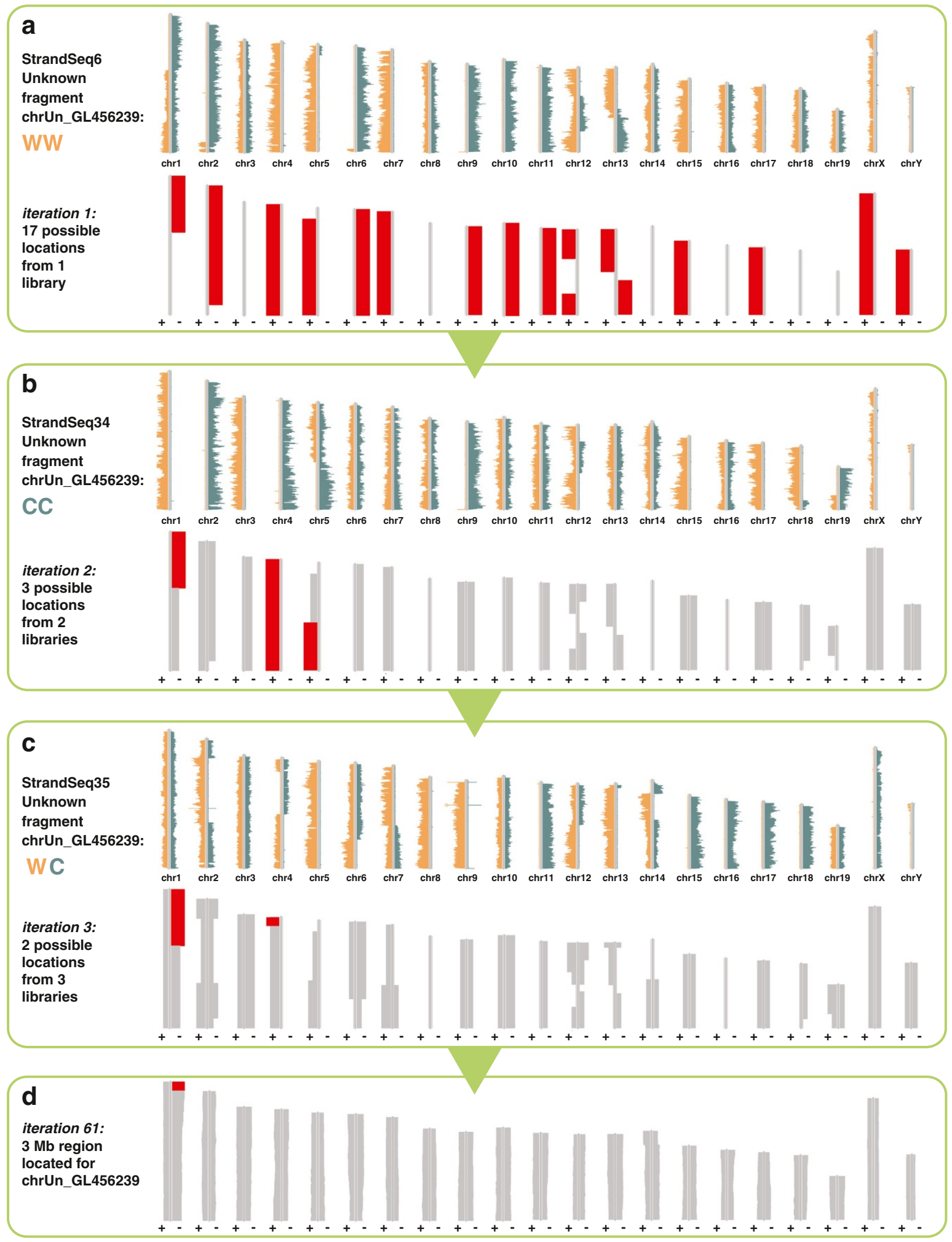

Figure 4 (See legend on next page.) 
(See figure on previous page.)

Figure 4 Bioinformatic Analysis of Inherited Templates (BAIT) localizes unplaced scaffolds in late-version assemblies. Orphan scaffolds can be correctly oriented and localized relative to the rest of the genome by comparing template-strand inheritance. The orientation of an orphan scaffold is arbitrary, because it is not anchored to the rest of the genome, so it can be correctly oriented with respect its located chromosome, or misoriented. (a) For a single library where the unplaced scaffold GL456239.1 is WW, BAIT maps its potential location (shown in red) to both WW genomic regions (correctly oriented), and CC genomic regions (misoriented). If only one library is analyzed, all locations map with 100\% concordance. Note that a WW scaffold will not locate to a WC chromosome, so chr8, chr14, chr16, chr18, and chr19 are 0\% concordant. (b) BAIT iterates over a second library where GL456239.1 is CC. The results of the two libraries combined reduce the number of potential mapping locations from 17 to only 3 that map with 100\% concordance. Because chr8, chr14, and chr16 are WC in this library also, these chromosomes map with 0\% concordance. (c) BAIT iterates over a third library where GL456239.1 is WC, and thus maps to all chromosomes that are WC. The result of the three combined libraries reduces the number of potential mapping locations to 2: the centromeric tips of chr 1 and chr4. (d) The combined results after iteration of all 62 libraries refine the location of GL456239.1 to the first $10 \mathrm{Mb}$ of chr 1 in the reverse orientation (with a concordance of 91\%). The fragment was further refined to an unbridged gap occupying the first 3 Mb of chr1. Abbreviations: C, Crick; chr, chromosome; W, Watson.

terminal regions of chromosomes are under-represented by BAIT's SCE localization. In addition, three of the SCE events predicted by BAIT but absent in the manual analysis were determined to be correct upon further analysis. One event was less than $2 \mathrm{Mb}$ from the distal telomere of chromosome 1, while the remaining two events were $5 \mathrm{Mb}$ from each other on chromosome 13 . These SCE events were difficult to detect by eye from a BAIT ideogram output of Strand-seq data. Furthermore, because BAIT identifies SCE locations directly on ideograms with an arrowhead, both false-positive and falsenegative SCEs can be rapidly scanned and validated from the ideogram output files.

Of the correctly identified SCE events, a comparison of the location of the SCE interval between automated and manual calls showed a median difference of just 34 bp (see Additional file 3: Figure S2). Almost two-thirds $(65.8 \%)$ of the predictions were within $100 \mathrm{bp}$ of the manual calls, with $74.7 \%$ of predictions within $10 \mathrm{~kb}$. A summary of SCE distribution across all libraries was plotted, together with a histogram reporting the distance between events, helping to identify significant clustering of SCEs (see Additional file 2: Supplemental Data File 1). The accurate identification of SCEs is also important for the functions of BAIT which assemble and refine reference genomes (see sections below).

BAIT facilitates SCE analyses by rapidly counting and locating events, presenting a pipeline that can be incorporated into high-throughput strategies. BAIT accurately refines the interval between reads in which the template switch occurs, allowing regions with a high propensity to undergo SCE to be identified (for example, fragile sites [26] or sites of recurrent DNA damage). Accurate interval identification is also important in looking for genomic rearrangements such as translocations, and BAIT is able to detect these and assign a frequency of the rearrangement within the pool of libraries, requiring a far lower read depth than conventional split-pair read sequencing [27]. A caveat to these analyses is that SCEs and genomic rearrangements are more difficult to detect on chromosomes that have more than two copies within a cell, potentially limiting its use in highly polyploid cancer cells. Taken together, our results show that BAIT is very accurate and efficient at predicting SCE intervals, and will be indispensable for future high-throughput analysis of Strand-seq data.

\section{Improving early-stage reference genome builds}

To test the ability of BAIT to build genomes de novo, we realigned our libraries to the first build of the mouse genome (MGSCv3). Of the 224,713 contigs in this assembly version, we included in the analysis the 77,258 that were over $10 \mathrm{~kb}$, representing 2,006 $\mathrm{Mb}$ of DNA (81.0\% of total assembly). After remerging and reorienting similar clusters, BAIT assigned 54,832 contigs, representing $1,742 \mathrm{Mb}(64.9 \%)$ of the assembly, into 20 primary LGs (Figure 3a). Allosomes in these male-derived ESCs are effectively monosome, and so contigs derived from the sex chromosomes can be separately identified, as they only inherit a single $\mathrm{W}$ or $\mathrm{C}$ template strand, never both. After cross-referencing the locations of MGSCv3 contigs to GRCm $38 / \mathrm{mm} 10$ coordinates, the majority of LGs clustered to only one chromosome (see Additional file 4: Figure S3), and the majority of chromosomes consisted of only one linkage group (Figure $3 \mathrm{~b}$ ). When more than one chromosome was attributed to the same linkage group, these groups could be split into two subclusters (see Additional file 4: Figure S3).

Similar results were seen when we simulated an early-stage reference by splitting the GRCm38/mm 10 genome into a scaffold of the 403 chromosomal Giemsa bands (based on coordinates from the UCSC genome browser [28]), and realigned our libraries to this new reference version (see Additional file 5: Figure S4). Using disrupted concordance from SCEs as a genetic distance indicator, it was further possible to infer the relative orders of the contigs present in each linkage group.

The accuracy of ordering fragments is dependent on the frequency of SCEs, the number of libraries used in 


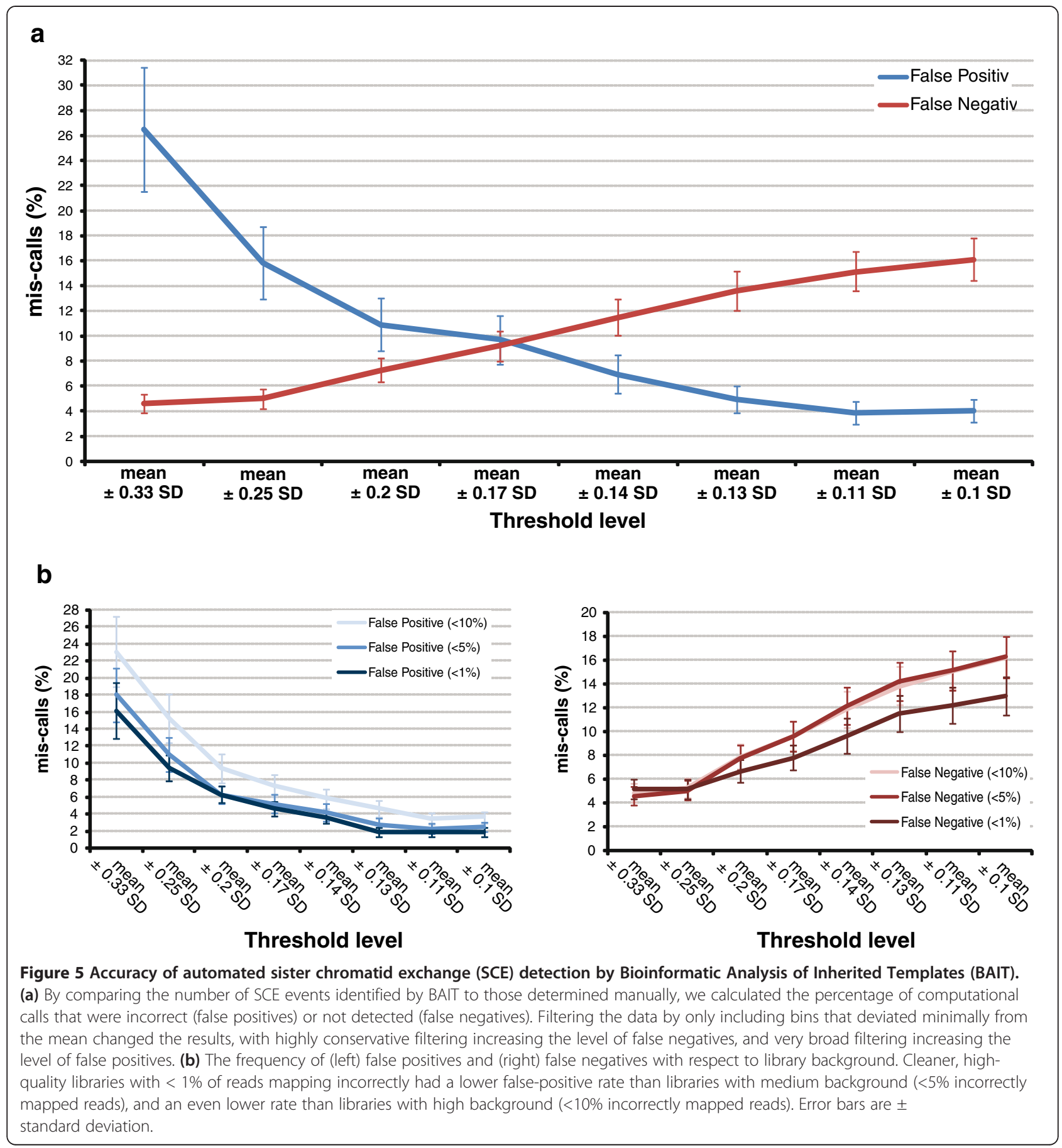

the analysis, and the level of library background (highbackground libraries are more likely to have incorrect template calls). If the template strands of contigs are identical in all libraries (because no SCE events have occurred between them) their relative order remains unknown.

Taken together, these data show that with only a single lane of sequencing and just 62 Strand-seq libraries, BAIT can aid in the rough draft assembly of a scaffold- level reference genome. Importantly, preliminary sequencing efforts in lesser-studied organisms suffer from fewer resources spent on deep sequencing and subsequent curating and refining of the reference genome assemblies. With several ambitious sequencing projects in development [13], there is an increasing need for rapid and cost-effective construction of accurate and useful reference genomes. Arranging contigs to facilitate building chromosome-level and genome-level hierarchy 
represents an attractive advance toward this goal, especially in conjunction with existing technologies. We have shown that BAIT can effectively 'stitch' contigs together based on shared template inheritance, and rapidly construct a useful skeleton assembly that can be built upon, and believe this technique will be widely adopted in standard genome assembly pipelines.

\section{Refining and finishing completed reference assemblies}

We have previously shown using Strand-seq that over 20 $\mathrm{Mb}$ of the MGSCv37/mm9 Mus musculus reference assembly is misoriented, involving 17 regions flanked by unbridged gaps [1]. In the more recent GRCm38/mm10 build of the genome, $35 \%(7,079.49 \mathrm{~kb})$ of these identified misorientations were subsequently corrected, validating Strand-seq with other approaches to correct orientation issues. In order to identify misorientations in the newest GRCm38/mm10 assembly, we repeated these analyses using the automated function of BAIT, identifying a total of 15 misoriented regions and 5 autosomal misorientations, with the remaining 10 located to the $\mathrm{X}$ chromosome (see Additional file 6: Table S1). Because the X chromosome only exists as one copy (monosomy) in the male embryonic stem cells (ESCs) of our dataset, misorientations appear indistinguishable from SCEs, and were identified by the intersection of events occurring over the same region across all libraries (see Additional file 2: Supplemental Data File 1). In this way, using just a single lane of sequencing, we were able to orient the majority of contigs (those larger than 10 $\mathrm{kb}$ with minimal segmental duplications) with respect to flanking contigs. Thus, using Strand-seq and BAIT with relatively low-coverage sequencing, the relative orientation of all reference contigs can be determined, effectively bridging all gaps in an assembly.

To validate the ability of BAIT to map scaffolds that have yet to be localized to regions on reference assemblies, we used it to predict the localization of all orphan scaffolds in an earlier assembly of the mouse reference (MGSCv37/mm9), and compared those predictions with the actual known locations in the current assembly (GRCm38/mm10). MGSCv37/mm9 has 60 useable orphan scaffolds that can be lifted to a single specific coordinate on GRCm38/mm10 [28]. Of these, 57 were located by BAIT to an interval coincident with the correct location on GRCm38/mm10 (Figure 6). From the three fragments that could not be correctly placed, two had fewer than 10 libraries with sufficient read counts to analyze, and the remaining fragment mapped with a low concordance (57.1\%). These data suggest reasonable thresholds for BAIT to map orphan scaffolds: more than 10 libraries and greater than 60\% concordance. More importantly, they confirm that using data from the same single lane of sequencing as used for contig orientation, BAIT and Strand-seq can correctly map a large proportion of orphan scaffolds in a late assembly version.

There remain 44 orphan scaffolds in GRCm38/mm10, accounting for 5,334,105 bp, and containing 41 known genes. Of these, 23 contained sufficient reads to analyze, and we were able to subsequently place all of them to their matching chromosomes to within narrow intervals (Table 1; see Additional file 7: Supplemental Data File 2). By intersecting these locations to gaps in the contiguous

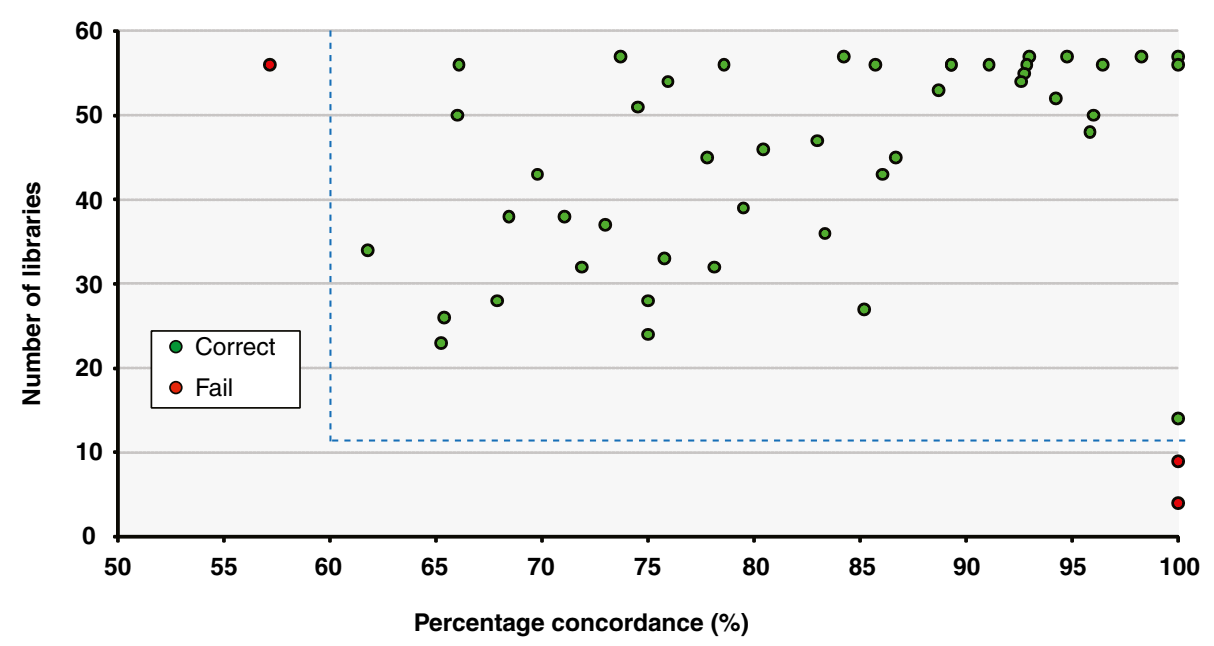

Figure 6 Validation of using Strand-seq to map unplaced scaffolds to built genomes. To confirm that Bioinformatic Analysis of Inherited Templates (BAIT) can successfully locate orphan scaffolds, the reads were aligned to MGSCv37/mm9, which has 202 orphan scaffolds, of which 60 can be mapped to a specific location in GRCm38/mm10. We used BAIT to locate these scaffolds in MGSCV37/mm9, and then cross-referenced these locations to the actual location in the GRCm38/mm10 assembly version. BAIT correctly located all regions in which there were more than 10 libraries to analyze, and where the percentage concordance was above 68\%. Green points indicate correctly mapped fragments, and red points indicate incorrectly mapped fragments. Dashed lines show the minimum number of libraries and minimal concordance needed to make confident calls 
Table 1 Locations of unplaced scaffolds on GRCm38/mm $10^{\mathrm{a}}$

\begin{tabular}{|c|c|c|c|c|c|c|c|c|c|}
\hline \multirow{2}{*}{$\begin{array}{l}\text { Accession } \\
\text { number }\end{array}$} & \multirow{2}{*}{$\begin{array}{l}\text { Scaffold } \\
\text { size, kb }\end{array}$} & \multirow{2}{*}{$\begin{array}{l}\text { Localizes } \\
\text { to: } \text {,Mb }^{\mathrm{b}}\end{array}$} & \multirow{2}{*}{$\begin{array}{c}\text { Strand } \\
\text { direction }^{\mathrm{b}}\end{array}$} & \multirow{2}{*}{$\begin{array}{c}\% \\
\text { Conc }^{c}\end{array}$} & \multirow{2}{*}{$\begin{array}{c}\text { Libraries }^{c}, \\
n\end{array}$} & \multicolumn{2}{|c|}{ Gaps $^{d}$} & \multicolumn{2}{|c|}{ Gap location ${ }^{\mathrm{e}}$} \\
\hline & & & & & & $(u), n$ & (b), $n$ & Primary & Alternate \\
\hline GL456382.1 & 23.2 & chrX:0-57.6 & + & 100 & 18 & 14 & 26 & & \\
\hline GL456379.1 & 72.4 & chrX:0-57.6 & - & 97.8 & 46 & 14 & 26 & & \\
\hline GL456233.1 & 336.9 & chrX:0-57.6 & - & 96.1 & 51 & 14 & 26 & & \\
\hline JH584299.1 & 953.0 & chr5:90.4-96.6 & + & 94.2 & 52 & 0 & 1 & chr5:94,088,336-94,138,335 & \\
\hline GL456239.1 & 40.1 & chr1:0-12.6 & - & 91.1 & 56 & 3 & 1 & chr1:0-3,000,000 & \\
\hline GL456367.1 & 42.1 & chrX:0-57.6 & - & 90.0 & 40 & 14 & 26 & & \\
\hline GL456381.1 & 25.9 & chrX:0-57.6 & - & 90.0 & 50 & 14 & 26 & & \\
\hline GL456393.1 & 55.7 & chr3:28.6-31.6 & + & 89.3 & 56 & 0 & 0 & chr3:40,550,618-40,650,617 & \\
\hline GL456359.1 & 23.0 & chr4:136.4-156.2 & - & 88.7 & 53 & 1 & 19 & chr4:156,408,117-156,508,116 & chr4:130,393,226-130,516,309 \\
\hline GL456354.1 & 196.0 & chr5:90.4-100.6 & - & 88.6 & 35 & 0 & 1 & chr5:94,088,336-94,138,335 & \\
\hline GL456385.1 & 35.2 & chr13:0-6.8 & - & 87.3 & 55 & 3 & 0 & chr13:1-3,000,000 & \\
\hline GL456360.1 & 31.7 & chr15:88.4-103.8 & + & 87.0 & 54 & 1 & 3 & $\operatorname{chr} 15: 103,943,686-104,043,685$ & \\
\hline GL456366.1 & 47.1 & chr15:62.4-103.8 & + & 85.5 & 55 & 1 & 3 & chr15:103,943,686-104,043,685 & \\
\hline GL456216.1 & 66.7 & chr4:136.4-156.2 & + & 80.4 & 51 & 2 & 19 & chr4:156,408,117-156,508,116 & chr4:130,393,226-130,516,309 \\
\hline$J H 584296.1$ & 199.4 & chr5:83.6-113.2 & - & 80.0 & 10 & 1 & 1 & chr5:113,521,975-113,535,974 & \\
\hline$J$ J584297.1 & 205.8 & chr5:88.6-100.6 & - & 77.8 & 18 & 0 & 1 & chr5:94,088,336-94,138,335 & \\
\hline GL456368.1 & 20.2 & chr4:129.2-156.2 & - & 76.2 & 42 & 2 & 19 & chr4:130,393,226-130,516,309 & chr4:156,408,117-156,508,116 \\
\hline GL456221.1 & 207.0 & chr1:79.8-123.2 & + & 73.7 & 57 & 2 & 4 & chr1:85,347,104-85,447,103 & chr1:75,055,557-75,121,556 \\
\hline GL456392.1 & 23.6 & chr2:0-8.2 & - & 73.5 & 34 & 4 & 0 & chr2:0-3,050,000 & \\
\hline JH584292.1 & 14.9 & chr4:107.8-108.6 & + & 73.5 & 49 & 0 & 1 & chr4:99,842,111-99,876,234 & \\
\hline GL456372.1 & 28.7 & chr1:127.2-146.2 & - & 69.2 & 52 & 1 & 1 & chr1:156,118,744-156,168,743 & \\
\hline GL456389.1 & 28.8 & $\operatorname{chr} X: 0-57.6$ & - & 63.6 & 33 & 14 & 26 & & \\
\hline GL456370.1 & 26.8 & chr4:67.6-68.8 & - & 62.0 & 50 & 0 & 3 & chr4:61,344,177-61,394,176 & \\
\hline
\end{tabular}

${ }^{\mathrm{O}}$ Of the 44 orphan scaffolds, 23 had enough reads to determine their genomic location by calculating mapping concordance.

${ }^{\mathrm{b}}$ The scaffold accession numbers and BAIT-determined locations are given, together with the strand direction, which gives the relative orientation of the scaffolds with respect to the genome.

'The percentage concordance (\% conc) and the number of libraries with enough information to make a concordance call are also given.

${ }^{d}$ Finally, BAIT cross-referenced these locations to unbridged and bridged gaps falling over the interval (gap (u) and gap (b) respectively.

ePrimary and alternate gap locations are given. To ensure no regions were missed, gaps were included within $10 \mathrm{Mb}$ away from the determined interval. 
genome build, BAIT further refined the scaffold locations (Table 1). Fragments were assumed to locate within either unbridged gaps or to bridged gaps in which gap size exceeded the fragment size, Analyzing 62 mouse libraries, $54.5 \%$ of these orphan scaffolds could be mapped to a particular chromosome, of which $54.2 \%$ could be mapped to a single contig gap (Table 1). BAIT also correctly oriented these fragments with respect to the chromosome to which they were mapped. For established and well-studied genomes, finishing builds by additional sequencing yields diminishing returns, and novel, targeted and highly sequence-efficient methodologies such as Strand-seq and BAIT can play a crucial role in completing these genomes. BAIT includes a utility to create a new FASTA reference genome by reverse complementing misoriented regions and incorporating orphan scaffolds that map to a defined gap.

\section{Conclusions}

BAIT provides the functionality to realize several powerful and exciting applications of Strand-seq: strand inheritance, SCE analysis, genomic rearrangements, and finishing genomes. With a robust strand-inheritance analysis tool and accurate SCE calling, BAIT is able to interrogate Strandseq data to follow template-strand segregation patterns, and is currently the most informative technique for testing such patterns [29-32]. In being able to identify SCE events to a kilobase resolution in one cell division (compared with a megabase resolution and two cell divisions for standard cytogenetic analysis [33,34]), Strand-seq offers a unique tool to examine regions of recurrent damage, and enumerates events in cells that have differing genetic backgrounds or have been subjected to different damaging agents. Crucially, these events can be independently assayed and mapped in individual chromosomes at a very high resolution without relying on cytogenetic expertise. In addition, we present here a novel use of template-strand analysis to localize fragments and orient contigs, which has yielded a more refined mouse reference assembly with $20.8 \mathrm{Mb}$ of contigs corrected (see Additional file 6: Table S1) and 2.7 Mb of orphan scaffolds localized to specific regions (Table 1). The ability to refine assemblies can be expanded to systematically stratify the thousands of scaffolds that make up earlyversion reference genome endeavors without the need for overlapping contigs to determine orientation or relative order. Taken together, BAIT will be indispensable for future Strand-seq studies, and we foresee its widespread adoption in a number of applications, most notably for refining and finishing assemblies at various levels of completeness.

\section{Availability and requirements}

- Project name: BAIT.

- Project homepage: See reference [15].
- Operating system: Linux.

- Programming language: BASH and R.

- Other requirements: SAMtools version 1.17 or higher, BEDtools version 2.17.0 or higher, $\mathrm{R}$ version 3.0 or higher, DNAcopy $\mathrm{R}$ package, gplots $\mathrm{R}$ package.

- License: Two-clause BSD.

- Restrictions for non-academics: license needed.

\section{Additional files}

Additional file 1: Figure S1. Flow chart of Bioinformatic Analysis of Inherited Templates (BAIT) pipeline. BAIT consists of a central Bash script involved in executing command line options and processing directional information from sequence data to be read into downstream R-scripts. Central decision options include generating contig orders from early build genomes, executing sister chromatid exchange (SCE) analysis and scanning the data for orphan scaffold alignment. Command-line options are shown as green labels adjacent to the arrows, with the input/output files represented as black rounded rectangles, and the graphical output files represented by grey rounded rectangles.

Additional file 2: Bioinformatic Analysis of Inherited Templates (BAIT) summary files, highlighting the locations of sister chromatid exchanges (SCEs) and misorientation events, as well as the minimal distances between SCE events across libraries. For interpreting template strand inheritance, pie charts and bin-level histograms are generated across all libraries being analyzed.

Additional file 3: Figure S2. Optimization of the sister chromatid exchange (SCE) interval detector function. After DNAcopy has identified the smallest region in which SCE events have occurred, Bioinformatic Analysis of Inherited Templates (BAIT) executes a function that scans the region from the homozygous template direction until it identifies the first read mapping to the opposite strand. These 'unfiltered calls' were similar to the manual calls, but were subject to low level background reads interfering with accurate localization (red line). To circumvent this, we added to the function that checks the 10 preceding reads to ensure they are all the same state, which yielded more accurate calls (blue line). Finally, we added a further check to ensure that the succeeding 20 reads (which are supposed to be Watson and Crick (WC)) mapped to the opposite strand at least $20 \%$ of the time (green line).

Additional file 4: Figure S3. Contig locations in Bioinformatic Analysis of Inherited Templates (BAIT)-compiled linkage groups. Strand-seq data was aligned to the MGSCV3 assembly, and was stratified solely on strand inheritance patterns. The resulting data were compared directly to the known locations of the MGSCv3 contigs in the current GRCm38/mm10 assembly. (a) Linkage groups determined by BAIT predominantly contain contigs derived from a single chromosome. The linkage groups (denoted as LG\# under each histogram) contain different numbers of clustered contigs, with the total length of each linkage group shown (y-axis) but tend to map to only one chromosome. (b) Of the linkage groups that map to more than one chromosome, a heatmap plot shows that the linkage group should be subdivided. Linkage group 1 (green highlight) contains contigs from chromosome 1 (chr1), chr15, and chr7, but generates three distinct clusters (left panel) where each cluster contains contigs derived from one chromosome (colours beneath dendrogram). An example of a linkage group with contigs mapping to a single locus (LG11, green highlight) shows that the majority of contigs within this group cluster tightly together (right panel).

Additional file 5: Figure S4. Validation of Strand-seq to cluster fragments to unanchored genomes. By measuring template concordance between mouse GRCm38/mm10 fragmented by chromosome banding pattern, a heat map was generated comparing the 403 scaffolds, of which 400 had enough reads across all libraries to accurately assign a template state. Scaffolds that inherited 
identical templates across all 62 libraries had a 'concordant distance' of 1.0 and are displayed as orange blocks. Because sister chromatid exchange (SCE) events change the template state in some libraries, the 'concordant distance' between contigs decreases, indicating that they are further apart. Scaffold orientation was determined through a greedy algorithm, and concordances were adjusted prior to plotting. To confirm that fragments were clustering correctly, their locations on the heat map were compared with the known chromosome (numbers below plot), showing a near-perfect clustering of scaffolds derived from the same chromosome. Three fragments had template states that appeared to occur independently of all the others (green boxes)

Additional file 6: Table S1. Identification of all misoriented fragments in $\mathrm{GRCm} 38 / \mathrm{mm} 10$. The genomic regions that were incorrectly oriented in the latest assembly version of the mouse genome were calculated by Bioinformatic Analysis of Inherited Templates (BAIT). The location and lengths of these regions, which should all be present in the reverse complement in the reference assembly, are shown. Misorientations were identified in every informative library.

\section{Additional file 7: Supplemental Data File 2 is the Bioinformatic} Analysis of Inherited Templates (BAIT) output of correlative data for all GRCm38/mm 10 unlocalized scaffolds. Standard output attempts to localize every genomic orphan scaffold by calculating the concordance of template strand inheritance across all libraries within each dataset (for an example, see Figure 4 for mapping of chrUn_GL456239). Each ideogram plot shows the likeliest location of the scaffold (red region), the percentage concordance (agreement between libraries), and the number of libraries used in the analysis.

\section{Abbreviations}

BAIT: Bioinformatic Analysis of Inherited Templates; BAM: Binary alignment map; BED: Browser Extensible Data; BrdU: 5-bromo-2'-deoxyuridine; BSD: Berkeley Software Distribution; CNV: Copy number variation; CSV: Comma-separated values; ESC: Embryonic stem cell; GRC: Genome Reference Consortium; LG: Linkage group; NCBI: National Center for Biotechnology Information; SCE: Sister chromatid exchange; UCSC: University of California Santa Cruz.

\section{Competing interests}

The authors declare that they have no competing interests.

\section{Authors' contributions}

$\mathrm{MH}$ wrote the paper, designed the figures, performed the analysis. and wrote the software. KO incorporated the SCE-identification function and the unknown fragment plotter into BAIT, and wrote the software. EF developed Strand-seq and helped to write the paper. RB helped with selection of computational approaches. PML conceived of the study and helped to write the paper. All authors read and approved the final manuscript.

\section{Acknowledgements}

We are grateful for advice and discussion from Ulrike Naumann, Ashley D Sanders, Elizabeth A Chavez, Duncan F Locke and Steven SS Poon. We thank Marianna Bevova for critical review of the manuscript. The Lansdorp laboratory is supported by grants from the Canadian Institutes of Health Research (RMF-92093 and 105265), the US National Institutes of Health (R01GM094146), and the Terry Fox Foundation (018006). PML is a recipient of an Advanced Grant from the European Research Council.

\section{Author details}

${ }^{1}$ Terry Fox Laboratory, BC Cancer Agency, Vancouver, BC V5Z 1L3, Canada. ${ }^{2}$ Division of Hematology, Department of Medicine, University of British Columbia, 675 West 10th Avenue, Vancouver, BC V5Z 1L3, Canada. ${ }^{3}$ European Research Institute for the Biology of Ageing, University of Groningen, University Medical Centre Groningen, A. Deusinglaan 1, NL-9713 AV Groningen, The Netherlands.

Received: 29 May 2013 Accepted: 9 September 2013

Published: 13 September 2013

\section{References}

1. Falconer E, Hills M, Naumann U, Poon SS, Chavez EA, Sanders AD, Zhao Y, Hirst M, Lansdorp PM: DNA template strand sequencing of single-cells maps genomic rearrangements at high resolution. Nat Methods 2012, 9:1107-1112.

2. Falconer $E$, Lansdorp PM: Strand-seq: a unifying tool for studies of chromosome segregation. Semin Cell Dev Biol 2013, 00068-2.

3. Aguilera A, Gomez-Gonzalez B: Genome instability: a mechanistic view of its causes and consequences. Nat Rev Genet 2008, 9:204-217.

4. Wilson DM 3rd, Thompson LH: Molecular mechanisms of sister-chromatid exchange. Mutat Res 2007, 616:11-23.

5. Wu L: Role of the BLM helicase in replication fork management. DNA Repair (Amst) 2007, 6:936-944.

6. Nagarajan N, Pop M: Sequence assembly demystified. Nat Rev Genet 2013, 14:157-167.

7. Pagani I, Liolios K, Jansson J, Chen IM, Smirnova T, Nosrat B, Markowitz VM, Kyrpides NC: The Genomes OnLine Database (GOLD) v. 4: status of genomic and metagenomic projects and their associated metadata. Nucleic Acids Res 2012, 40:D571-D579.

8. Waterston RH, Lindblad-Toh K, Birney E, Rogers J, Abril JF, Agarwal P, Agarwala R, Ainscough R, Alexandersson M, An P, et al: Initial sequencing and comparative analysis of the mouse genome. Nature 2002, 420:520-562.

9. Tettelin H, Radune D, Kasif S, Khouri H, Salzberg SL: Optimized multiplex PCR: efficiently closing a whole-genome shotgun sequencing project. Genomics 1999, 62:500-507.

10. Samad A, Huff EF, Cai W, Schwartz DC: Optical mapping: a novel, singlemolecule approach to genomic analysis. Genome Res 1995, 5:1-4.

11. Marra MA, Kucaba TA, Dietrich NL, Green ED, Brownstein B, Wilson RK, McDonald KM, Hillier LW, McPherson JD, Waterston RH: High throughput fingerprint analysis of large-insert clones. Genome Res 1997, 7:1072-1084.

12. Levy-Sakin M, Ebenstein Y: Beyond sequencing: optical mapping of DNA in the age of nanotechnology and nanoscopy. Curr Opin Biotechnol 2013 24:690-698.

13. Genome 10K Community of Scientists: Genome 10K: a proposal to obtain whole-genome sequence for 10,000 vertebrate species. J Hered 2009, 100:659-674.

14. Bernardi G, Wiley EO, Mansour H, Miller MR, Orti G, Haussler D, O'Brien SJ, Ryder OA, Venkatesh B: The fishes of Genome 10K. Mar Genomics 2012, 7:3-6.

15. BAIT. http://sourceforge.net/p/bait/wiki/Home/

16. Li H, Handsaker B, Wysoker A, Fennell T, Ruan J, Homer N, Marth G, Abecasis G, Durbin R: The Sequence Alignment/Map format and SAMtools. Bioinformatics 2009, 25:2078-2079.

17. Gentleman RC, Carey VJ, Bates DM, Bolstad B, Dettling M, Dudoit S, Ellis B, Gautier L, Ge Y, Gentry J, et al: Bioconductor: open software development for computational biology and bioinformatics. Genome Biol 2004, 5:R80-R80.16.

18. Quinlan AR, Hall IM: BEDTools: a flexible suite of utilities for comparing genomic features. Bioinformatics 2010, 26:841-842.

19. Holm S: A Simple sequentially rejective multiple test procedure. Scand J Stat 1979, 6:65-70.

20. Olshen $A B$, Venkatraman $E S$, Lucito $R$, Wigler M: Circular binary segmentation for the analysis of array-based DNA copy number data. Biostatistics 2004, 5:557-572.

21. Seshan VE, Olshen A: DNAcopy: DNA copy number data analysis. R package version 1.16.0. 2010. http://www.bioconductor.org/packages/2.3/bioc/html/ DNAcopy.html.

22. Copeland NG, Jenkins NA: Development and applications of a molecular genetic linkage map of the mouse genome. Trends Genet 1991, 7:113-118.

23. Croes GA: A method for solving traveling-salesman problems. Oper Res 1958, 6:791-812.

24. Nagarajan N, Cook C, Di Bonaventura M, Ge H, Richards A, Bishop-Lilly KA, DeSalle R, Read TD, Pop M: Finishing genomes with limited resources: lessons from an ensemble of microbial genomes. BMC Genomics 2010, 11:242-251.

25. Kent WJ, Sugnet CW, Furey TS, Roskin KM, Pringle TH, Zahler AM, Haussler D: The human genome browser at UCSC. Genome Res 2002, 12:996-1006.

26. Sutherland GR, Baker E, Seshadri RS: Heritable fragile sites on human chromosomes. V. A new class of fragile site requiring BrdU for expression. Am J Hum Genet 1980, 32:542-548. 
27. Chen W, Kalscheuer V, Tzschach A, Menzel C, Ullmann R, Schulz MH, Erdogan F, Li N, Kijas Z, Arkesteijn G, et al: Mapping translocation breakpoints by next-generation sequencing. Genome Res 2008, 18:1143-1149

28. Meyer LR, Zweig AS, Hinrichs AS, Karolchik D, Kuhn RM, Wong M, Sloan CA, Rosenbloom KR, Roe G, Rhead B, et al: The UCSC Genome Browser database: extensions and updates 2013. Nucleic Acids Res 2013, 41:D64-D69.

29. Cairns J: Mutation selection and the natural history of cancer. Nature 1975, 255:197-200.

30. Potten CS, Hume WJ, Reid P, Cairns J: The segregation of DNA in epithelial stem cells. Cell 1978, 15:899-906.

31. Lansdorp PM: Immortal strands? Give me a break. Cell 2007, 129:1244-1247.

32. Falconer E, Chavez EA, Henderson A, Poon SS, McKinney S, Brown L, Huntsman DG, Lansdorp PM: Identification of sister chromatids by DNA template strand sequences. Nature 2010, 463:93-97.

33. Kato H: Spontaneous sister chromatid exchanges detected by a BUdRlabelling method. Nature 1974, 251:70-72.

34. Allen JW, Latt SA: Analysis of sister chromatid exchange formation in vivo in mouse spermatogonia as a new test system for environmental mutagens. Nature 1976, 260:449-451.

\section{Submit your next manuscript to BioMed Central and take full advantage of:}

- Convenient online submission

- Thorough peer review

- No space constraints or color figure charges

- Immediate publication on acceptance

- Inclusion in PubMed, CAS, Scopus and Google Scholar

- Research which is freely available for redistribution
( BioMed Central 